\title{
An artificial neural network based fast radiative transfer model for simulating infrared sounder radiances
}

\author{
Praveen Krishnan, K Srinivasa Ramanujam and C Balaji* \\ Indian Institute of Technology Madras, Chennai 600 036, India. \\ *Corresponding author. e-mail: balaji@iitm.ac.in
}

The first step in developing any algorithm to retrieve the atmospheric temperature and humidity parameters at various pressure levels is the simulation of the top of the atmosphere radiances that can be measured by the satellite. This study reports the results of radiative transfer simulations for the multichannel infrared sounder of the proposed Indian satellite INSAT-3D due to be launched shortly. Here, the widely used community software k Compressed Atmospheric Radiative Transfer Algorithm (kCARTA) is employed for performing the radiative transfer simulations. Though well established and benchmarked, kCARTA is a line-by-line solver and hence takes enormous computational time and effort for simulating the multispectral radiances for a given atmospheric scene. This necessitates the development of a much faster and at the same time, equally accurate RT model that can drive a real-time retrieval algorithm. In the present study, a fast radiative transfer model using neural networks is proposed to simulate radiances corresponding to the wavenumbers of INSAT-3D. Realistic atmospheric temperature and humidity profiles have been used for training the network. Spectral response functions of GOES-13, a satellite similar in construction, purpose and design and already in use are used. The fast RT model is able to simulate the radiances for 1200 profiles in $18 \mathrm{~ms}$ for a 15-channel GOES profile, with a correlation coefficient of over $99 \%$. Finally, the robustness of the model is tested using additional synthetic profiles generated using empirical orthogonal functions (EOF).

\section{Introduction}

Remote sensing of the earth's atmosphere has been a subject of interest for several decades for many researchers from diversified areas. The parameters of interest related to earth's atmosphere to be retrieved from satellite observations, include temperature of earth's atmosphere at various pressure levels and humidity. The first step in the inverse analysis of radiation is to model the radiative heat transfer emerging from the earth's surface and passing through the atmosphere consisting of mixture of several gases. The latter can emit and absorb the radiation in the infrared region, and hence is participatory in nature. Hence, an algorithm needs to be developed in order to simulate the radiances (or brightness temperature by inverting the Planck's function) that would be measured by a typical sounder for different wavenumbers. The process that facilitates this simulation of infrared radiances for a given set of atmospheric parameters is known as the forward model. The forward model is then coupled with an optimization tool to accomplish the retrievals.

Many studies on infrared-based retrievals are available. In literature, one of the earliest works in the retrieval of temperature profile was done by Smith et al. (1972). In this work, the authors have

Keywords. Radiative transfer; infrared; multi spectral; INSAT 3D; neutral network. 
used a dynamic forecasting algorithm using data from Nimbus IV Satellite Infrared Spectrometer. This algorithm measures the deviations from an initial guess profile, and iteratively minimizes the radiance residues.

Yeh et al. (1985) developed an inversion method to retrieve temperature profiles under any cloudy situation and several case studies were done with data obtained from NOAA-6 and TIROS-N High Resolution Infrared Sounder (HIRS/2). Liou et al. (1992) proposed a direct inversion (DI) method that avoids the need for a first guess profile. This method uses convolution theory and applies Laplace transforms to arrive at temperature profile determination.

Li (1994) applied a one term variational method for the RTE, to determine the successive form of temperature weighting functions by considering surface emissivity and solar reflectivity. Geostationary Operational Environmental Satellite (GOES)-8 and GOES-9 sounders were launched to retrieve atmospheric temperature and water vapour profiles and the results are documented in this study.

Among the most recent studies, Machado et al. (1998) presented a new technique for the fast computation of near monochromatic atmospheric transmittances in the infrared, utilizing compressed look-up tables, that is well suited for nadir viewing satellite and airplane observations. A Singular Value Decomposition (SVD) technique was used to transform very large monochromatic look-up tables of absorption coefficients into a compressed representation that is almost 100 times smaller. This technique became the basis of the radiative transfer solver $\mathrm{k}$ Compressed Atmospheric Radioactive Transfer Algorithm(kCARTA) developed by the same team.

Andrieu and Arnaud Doucet (2000) presented a simulated annealing based approach for maximum a posteriori parameter estimation of Hidden Markov Models (HMM). Crewell et al. (2002) developed a synergetic algorithm which incorporates measurements from various instruments in comparison with other retrieval algorithms. The comparison of different retrieval methods reveals the weakness of neural network retrievals in unusual situations.

Suggs et al. (2003) emphasize that the knowledge of surface emissivity in the GOES Imager and Sounder infrared channels that receive energy from the earth's surface is necessary for an accurate retrieval of the atmospheric temperature and moisture profiles. The authors further observed that the Land surface temperature (LST) and total precipitable water (TPW) retrievals are especially sensitive to emissivity assumptions because they rely almost exclusively on these window channel measurements. In order to retrieve these atmospheric and surface geophysical parameters, the authors have considered a priori estimates of surface emissivity for forward radiative transfer calculations. However, the surface is assumed to be a gray-body with a constant emissivity (spatially and for all channels) in their calculations.

Jiang et al. (2005) and Blackwell (2005) show the use of neural networks for the retrieval of temperature and moisture profiles from the radiances of the high spectral sounding measurements. This strengthens the belief that Artificial Neural Network can work as a good retrieval tool.

There were many Indian satellite missions in the past. However, unlike previous geostationary meteorological satellites launched by the Indian Space Research Organisation (ISRO), the INSAT$3 \mathrm{D}$ which is due for launch in 2012, will permit programmable scanning of a special sector, with defined N-S and E-W coordinates. Scanning will also be faster and the data will be at a higher resolution compared to the currently operational satellites (Katti et al. 2006). In view of this, the geophysical products derived during the scanning will be more accurate and of higher resolution. The proposed INSAT-3D satellite scans the earth's atmosphere in 19 channels whose wavenumbers are similar to the Geostationary Operational Environmental Satellite (GOES) launched by National Oceanic and Atmospheric Administration (NOAA). The GOES has 19 different channels of which one is an imager and three channels are suited for the cloudy sky conditions (CIMSS GOES 13 science report 2006). Since wavenumbers corresponding to the infrared sounder of INSAT$3 \mathrm{D}$ are similar to that of GOES, the wavenumbers corresponding to the GOES are used in the present study.

In order to carry out the forward calculations, a freely distributed and widely used kCARTA has been used that depicts the physics as seen by a satellite. The layer averaged thermodynamic properties of the participating gases in the atmosphere such as pressure, humidity, temperature, together with the concentrations of the trace gases at various altitudes such as carbon dioxide, methane and ozone constitute the input. kCARTA is used to solve the radiative transfer equation under clear sky conditions. Clear sky condition simulations are carried out since the earth's atmosphere is opaque in cloudy conditions when scanned using an IR sounder. The output from the kCARTA contains the simulated values of the radiances over a wavenumber ranging from 630 to $2680 \mathrm{~cm}^{-1}$ with an interval of $0.0025 \mathrm{~cm}^{-1}$. Hence, for a given set of temperature and humidity conditions in the atmosphere, kCARTA simulates 820,000 radiances at their corresponding wavenumbers. 
As mentioned earlier, of the 19 channels on GOES, one is an imager and three channels are suited for cloudy sky conditions. Hence, in the present study which involves multispectral infrared sounder, only 15 channels are considered. The GOES measures the radiances at these 15 chosen wavenumbers, however the kCARTA simulates radiances at 820,000 wavenumbers for the same range. Hence, in the present study, radiances at wavenumbers corresponding to the 15 channels of the GOES sounder instrument are simulated. The instrument's response for the variation in intensity at a wavelength $\lambda$ about $d \lambda$ is known as the Spectral Response Function. In the present work SRFs have also been accounted. The radiances obtained from kCARTA are integrated over the SRF in the given wavenumber range. A large database is generated by simulating the radiances using kCARTA for the temperature and humidity profiles measured from five different sources. Finally, a much faster algorithm based on neural network is proposed to speed up the calculations involved in the simulations of the infrared radiances. This is necessitated by the requirement of repeated calculations of brightness temperatures for accomplishing the retrievals.

\section{The forward model}

The Radiative Transfer Equation (RTE) is the governing equation for the propagation of electromagnetic radiation through an absorbing, emitting and scattering medium. In the present work, we consider the propagation of electromagnetic radiation in the infrared range to compute the radiances or brightness temperatures leaving the top of the atmosphere at various infrared frequencies for a given atmospheric state, as shown in figure 1. Since the focus of the work is on developing a fast radiative transfer model for clear sky conditions, scattering terms in the RTE are neglected.

The intensity of the radiation emerging from the earth's surface and passing through an emitting and absorbing medium is given by the RTE;

$$
\mu \frac{d I_{\eta}}{d \tau}=-\kappa_{\eta} I_{\eta}+\varepsilon_{\eta} I_{b \eta}
$$

In equation (1), $I$ is the diffuse radiance field, $\kappa$ is the absorptivity, $\varepsilon$ is the emissivity and $\tau$ is the optical depth. The cosine of the zenith angle $\theta$ is denoted as $\mu$ and $\eta$ refers to wavenumber. The first term on the right hand side of equation (1) represents the attenuation in the intensity of outgoing radiation due to absorption by the participating gases. The second term represents the augmentation of intensity due to emission by the participating gases by virtue of its temperature. $I_{b \eta}$ is the spectral intensity of the black body given by the Planck's function in terms of wavenumber and temperature as:

$$
I_{b \eta}=\frac{c_{1} \eta^{5}}{\exp \left(c_{2} \eta / T\right)-1}
$$

where $c_{1}$ and $c_{2}$ are first and second radiation constants, respectively.

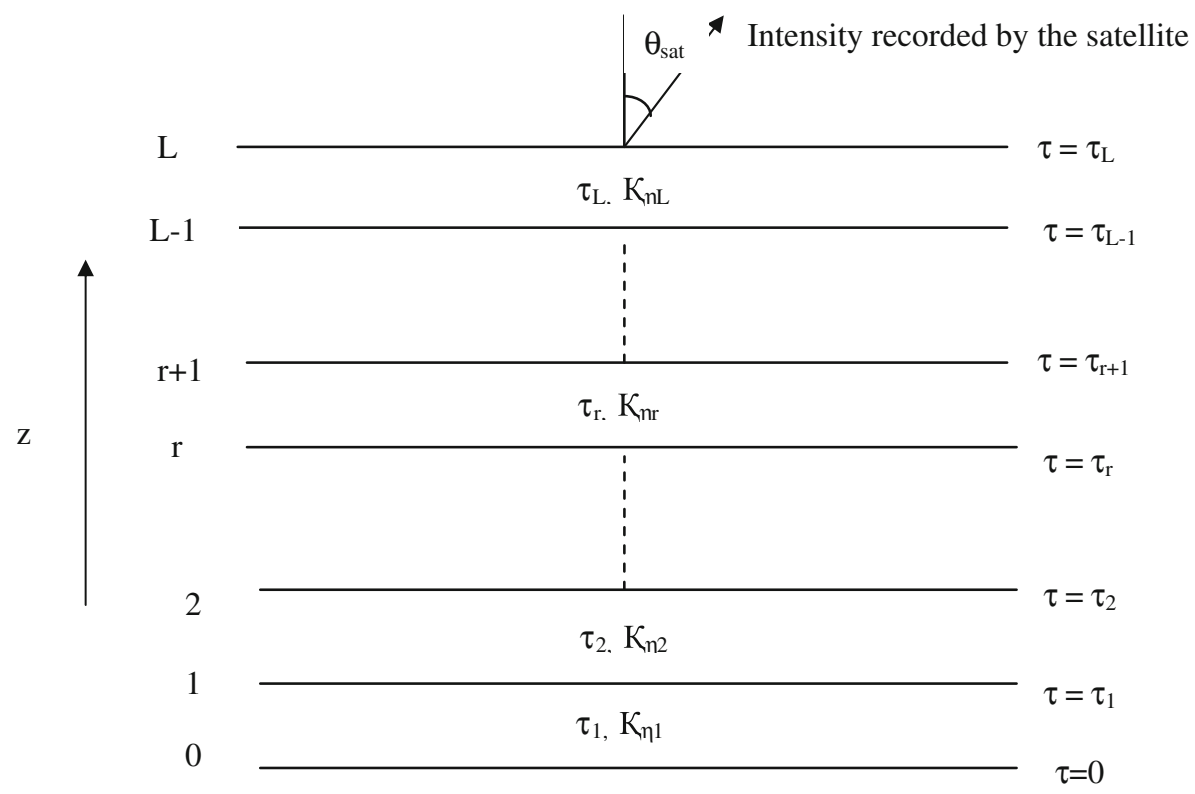

Figure 1. Schematic of the layered atmosphere model without scattering. 
Top of atmosphere

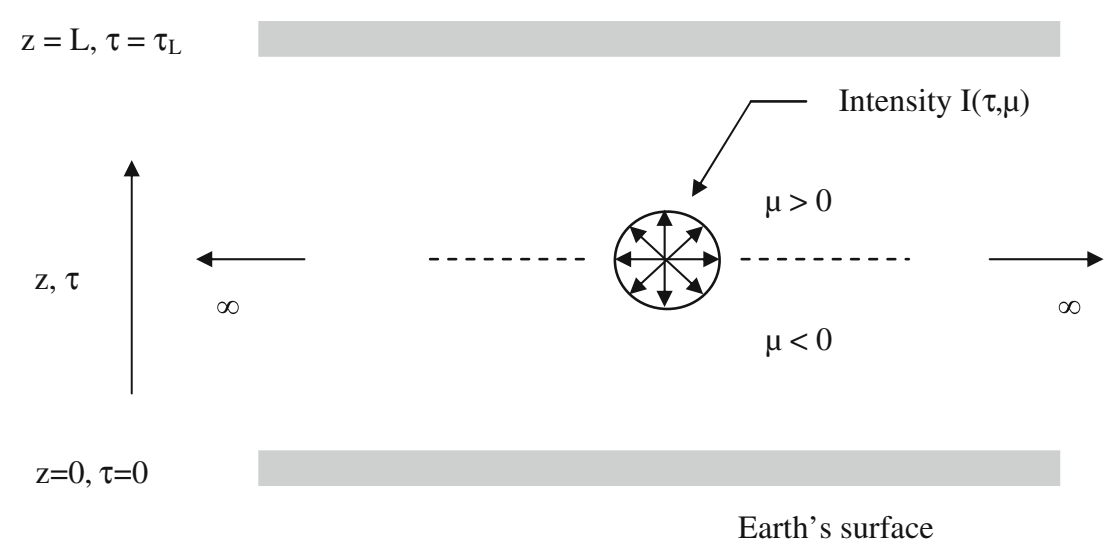

Figure 2. Schematic of a plane parallel medium.

Following the Kirchoff's law for an isothermal medium and substituting for $I_{b \eta}$, the radiative transfer equation becomes:

$$
\mu \frac{d I_{\eta}}{d \tau}=-\kappa_{\eta} I_{\eta}+\kappa_{\eta} \cdot \frac{c_{1} \cdot \eta^{5}}{\exp \left(c_{2} \eta / T\right)-1} .
$$

Figure 2 shows the one dimensional inhomogeneous atmosphere with a participating medium of thickness $L$ bounded by two parallel surfaces at different temperatures. The bottom surface represents the ocean surface characterized by a sea surface temperature given by:

$$
T_{s}=T_{1}+\rho \sigma
$$

where $T_{S}$ is the sea surface temperature, $T_{1}$ is the temperature at the first level, ' $\rho$ ' is a random number with 0 mean, and ' $\sigma$ ' is the standard deviation which is assumed to be constant, 2 K. For computational purposes, the vertical atmosphere is divided into 96 layers of varying thickness in which the atmosphere is considered to be homogeneous. The participating medium is considered to be non-gray.

An exact solution exists for the RTE given by equations (1-3) under clear sky conditions. However, in order to solve the RTE, the optical thicknesses of the different layers and absorptivity of various gases at the different wavenumbers need to be known accurately. In the present work, the open source Fortran Code called kCARTA was used for solving the RTE. The kCARTA software uses look-up tables for determining the absorptive coefficients of the various gases involved. The lookup tables are compressed using a Singular Value Decomposition (SVD) technique, to produce the kCompressed database. The point spacing of the current database is $0.0025 \mathrm{~cm}^{-1}$, which is an average over five points spaced at $0.0005 \mathrm{~cm}^{-1}$. To compute the absorption coefficients for a given profile, the look-up tables are spline (cubic) interpolated in temperature, and scaled in gas absorber amount. The software is set to produce the radiances over a wavenumber span ranging from 630 to $2800 \mathrm{~cm}^{-1}$. Radiances are simulated at one hundred pressure layers from 1100 to 0.005 mbar. The pressure layers considered in present study are similar to the pressure levels used for the Atmospheric Infrared Sounder (AIRS) Fast Forward Model, for which kCARTA is used as the 'Reference Forward Model'. The gases considered in the present analysis are the trace gases namely carbon dioxide, ozone, water vapour and methane. The concentration of carbon dioxide is kept constant while all other radiatively significant gases have variable concentrations.

The input profile is first processed through KLAYER, a function which converts point profiles to layer averaged temperature profiles. The KLAYER routine converts the atmospheric profile to Radiative Transfer Profile (RTP) format which is compatible with kCARTA program. This RTP file along with the name list containing the wavenumber range and emissivity information is input into the main program of kCARTA which calculates the radiances in the infrared region as specified.

In the present study, simulations are carried out at wavenumbers corresponding to the GOES Sounder instrument to mimic the 'measurements' of the radiances leaving the top of the atmosphere (TOA). Table 1 gives the details about the channels that are considered in the present study.

\subsection{Spectral response functions}

Spectral response functions (SRF) show the behaviour of an instrument at a particular wavenumber $v$ about $d v$. Figure 3 shows the 18 
Table 1. Channels and their details used for simulations in the fast forward model.

\begin{tabular}{lccl}
\hline $\begin{array}{l}\text { Channel } \\
\text { no. }\end{array}$ & $\begin{array}{c}\text { Wavelength } \\
(\mu \mathrm{m})\end{array}$ & $\begin{array}{c}\text { Wavenumber } \\
\left(\mathrm{cm}^{-1}\right)\end{array}$ & $\begin{array}{c}\text { Principal absorbing } \\
\text { constituents }\end{array}$ \\
\hline 1 & 14.71 & 680 & $\mathrm{CO}_{2}$ band \\
2 & 14.37 & 696 & $\mathrm{CO}_{2}$ band \\
3 & 14.06 & 711 & $\mathrm{CO}_{2}$ band \\
4 & 13.96 & 733 & $\mathrm{CO}_{2}$ band \\
5 & 13.37 & 748 & $\mathrm{CO}_{2}$ band \\
6 & 12.66 & 790 & Water vapour \\
7 & 12.02 & 832 & Water vapour \\
8 & 11.03 & 907 & Window \\
9 & 9.71 & 1030 & Ozone \\
10 & 7.43 & 1345 & Water vapour \\
11 & 7.02 & 1425 & Water vapour \\
12 & 6.51 & 1532 & Water vapour \\
13 & 4.57 & 2188 & $\mathrm{~N}_{2}, \mathrm{O}$ \\
14 & 4.52 & 2213 & $\mathrm{~N}_{2}, \mathrm{O}$ \\
15 & 4.45 & 2247 & $\mathrm{CO}_{2}$ \\
\hline
\end{tabular}

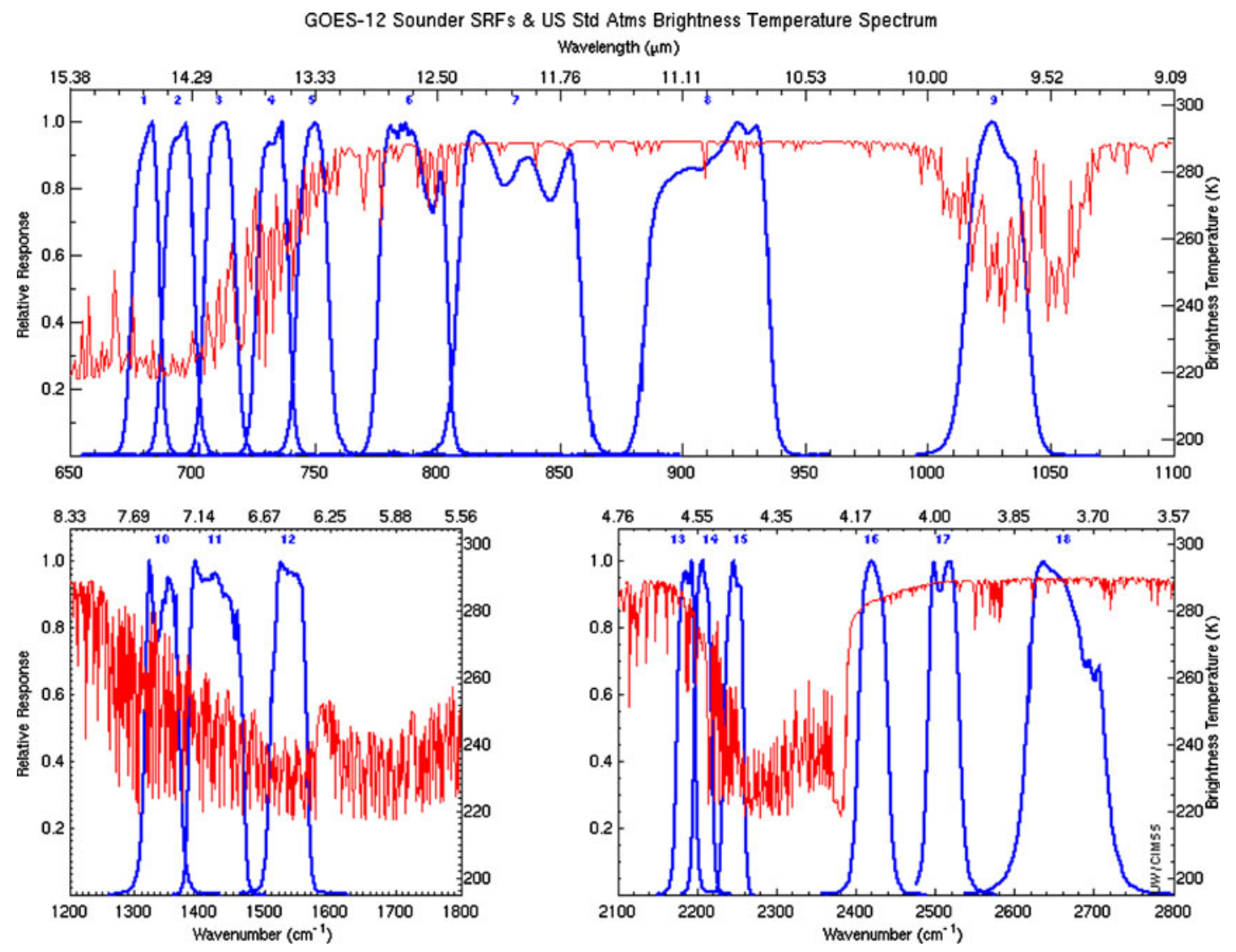

Figure 3. The SRFs for the 18 channels of the GOES infrared sounder (reproduced from CIMSS GOES 12 science report 2006).

SRFs corresponding to the 18 wavenumbers of GOES on the electromagnetic spectrum. In the present work, it is assumed that the SRFs of INSAT-3D closely match those of GOES. The SRFs for the first 15 channels are considered in the present analysis which are obtained from the
Donald and Timothy (2007). The SRF for a particular channel is incorporated as:

$$
\int_{0}^{\infty} F(v) B\left(v, T_{e}\right) d v=\int_{0}^{\infty} F(v) R(v) d v
$$


where $F$ is the SRF, $B$ is the Planck's black body intensity for a given temperature $T_{e}$ and $R$ is the intensity of emitted energy at wavenumber $v$ measured by the instrument. Since the interval of wavenumber is the same on both sides of the equation, the convolved radiance turns out to be:

$$
B=\frac{\sum F(\nu) \cdot R(\nu)}{\sum F(\nu)} .
$$

\section{Need for the fast forward model}

Forward calculations are an integral part of any retrieval problem. Retrieval algorithms can be broadly classified into two types: (i) statistics based and (ii) physics based. Physics-based retrieval algorithms are widely used in remote sensing applications due to their robustness and their ability to solve the non-linear inversion problem, particularly for temperature and water vapour profile retrieval.

Physics-based algorithms suffer from the drawback that it is necessary to execute the forward model repeatedly for each retrieval in order to find the 'best' profile that matches with the satellite observations. Therefore, the time involved in the forward calculations becomes critical in the retrieval algorithm. However, the computation time involved in simulating the radiances using kCARTA is very high since kCARTA is a line-byline method. It has been observed that it takes about $58 \mathrm{~s}$ to simulate the radiances at different wavenumbers for a given atmospheric scene on an Intel Xeon based Linux machine. However, infrared sounders typically make a measurement of about 16 pixels or profiles every second per orbit (Donald and Timothy 2007). Hence, in order to develop an algorithm to retrieve the atmospheric profiles, there is a critical need to speed up the computational time involved in the forward calculations for real time processing of satellite images with a view to estimate the vertical atmospheric structure. Hence, a fast, accurate and robust radiative transfer model to simulate the radiances in the atmosphere becomes a necessity. In the present work, an artificial neural network (ANN) based fast forward model is proposed to replace the kCARTA.

\section{Artificial neural networks (ANN) based fast forward model (AFFM)}

Neural networks have been a simple and effective technique to establish a relationship between the input and the corresponding target values. In the present study, an ANN based fast forward model (AFFM) is proposed to speed up the forward calculations. A simple neural network architecture is shown in figure 4. The input layer consists of 193 neurons corresponding to the 96 temperatures and 96 humidity parameters along with the sea surface temperature, while the output layer has 15 neurons corresponding to the 15 brightness temperatures. One hidden layer is used for training the network in the present study. Statistics based methods such as ANN are largely data driven and hence the generation of database for retrievals forms a very crucial step.

\subsection{Generation of database}

In the present study, vertical profiles are considered at 96 layers of the atmosphere between the surface of the earth and up to a height of $80 \mathrm{~km}$. The realistic database consists of layerwise information for temperature, humidity, ozone

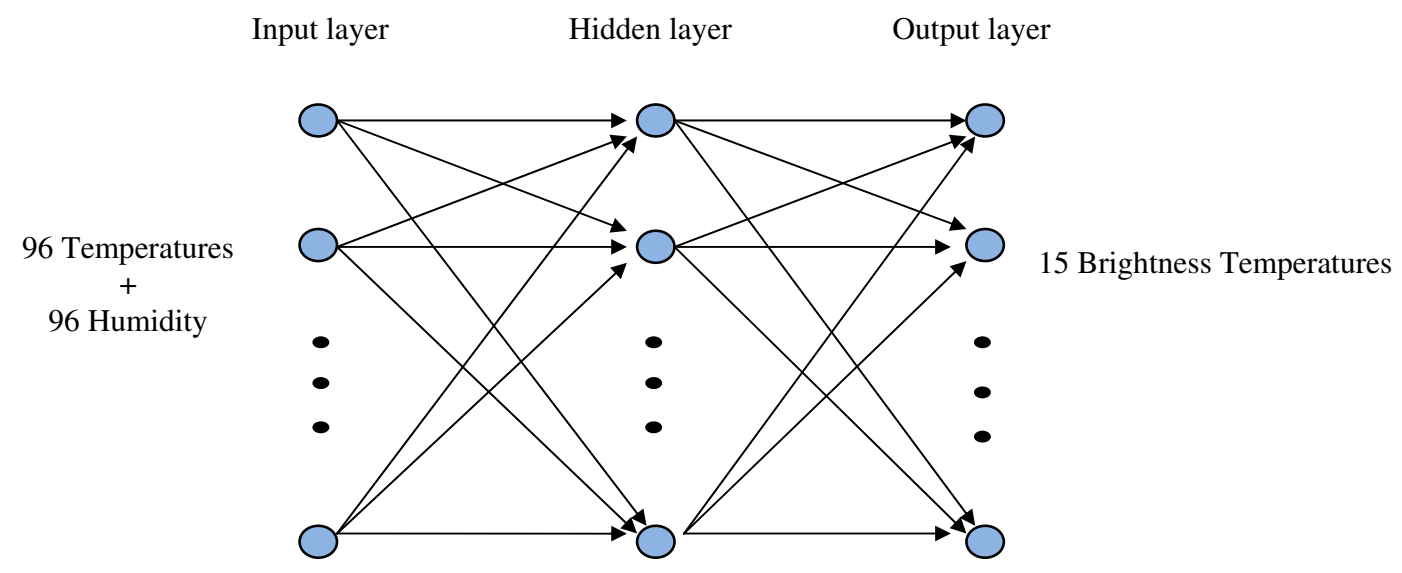

Figure 4. A simple neural network architecture of the forward model depicting the input layer, output layer and the hidden layer. 


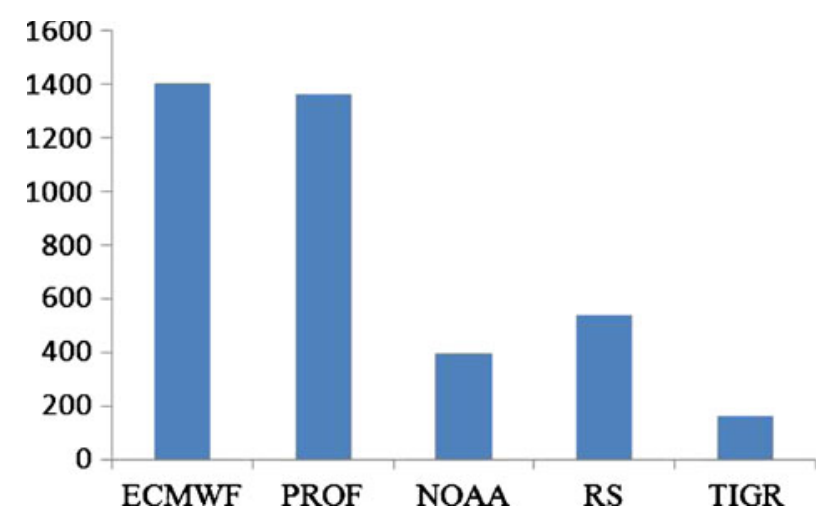

Figure 5. Distribution of realistic database of atmospheric temperature and humidity profiles.

and gaseous constituents such as carbon dioxide, methane and ozone. Temperature and humidity profile data from NOAA, Thermodynamic Initial Guess Retrieval (TIGR), RadioSonde, OzoSonde and European Centre for Medium Range Forecasts (ECMWF) are considered to develop the fast forward model. The total number of profiles collected from these sources amounts to 11,450. A special request was placed to Laboratoire de Meteorologie Dynamique (LMD), France in order to obtain this data. Figure 5 shows the distribution of the realistic database. Of these 11,450 profiles, 3876 profiles corresponding to the tropical region bounded by $\pm 25^{\circ}$ latitude alone are considered in this study.

An a priori database is generated by executing the kCARTA for the 3876 profiles. The datasets are classified into two sets: the first set of data consisting of 2676 profiles is used for training the network while the remaining are used for testing purposes. The input consists of 193 parameters; 96 values of temperatures and 96 values of humidity along with the sea surface temperature at various vertical levels in the atmosphere. The output consists of 15 brightness temperatures simulated by the kCARTA for the corresponding input profiles. The inputs and the outputs are normalized against their respective maximum and minimum values before they are input to the network with the following expression:

$$
P_{n}(i)=\frac{P(i)-P_{\min }}{P_{\max }-P_{\min }}
$$

where $P$ is either input or output parameter, $P_{n}(i)$ refers to the normalized parameter, $P_{\max }$ and $P_{\min }$ refer to the maximum and minimum values of the parameters, respectively. The back propagation neural network toolbox available in the commercial package MATLAB is used for training and simulation of the network.

\section{Generation of synthetic profiles}

In the present study, apart from the need to expand the existing database, the generation of synthetic profiles is also necessitated to analyze the robustness of the fast forward model proposed. The initial dataset of radiosonde profiles are decomposed using the method of Empirical Orthogonal Functions (EOF) to generate the synthetic database. The corresponding eigen profiles and the statistics of the expansion coefficients are used to numerically generate synthetic profiles that obey the same statistics (with the same mean, variability and cumulative eigenvectors) as the initial dataset.

The temperature and humidity profiles are generated separately using EOF. Stochastic temperature profiles are numerically generated using the following equation (Tatarskaia et al. 1997)

$$
\begin{gathered}
T\left(z_{k}\right)=\left\langle T\left(z_{k}\right)\right\rangle+\sum_{v=1}^{96} \zeta_{v} \phi_{v}^{T}\left(z_{k}\right) \sqrt{\lambda_{v}^{T}} . \\
k=1,2, \ldots, 96
\end{gathered}
$$

where the superscript $T$ identifies temperature quantities, the angle brackets $\langle\cdots\rangle$ describe an average over the ensemble of radiosonde profiles in a particular classification, $\phi_{v}^{T}\left(z_{k}\right)$ is the eigen
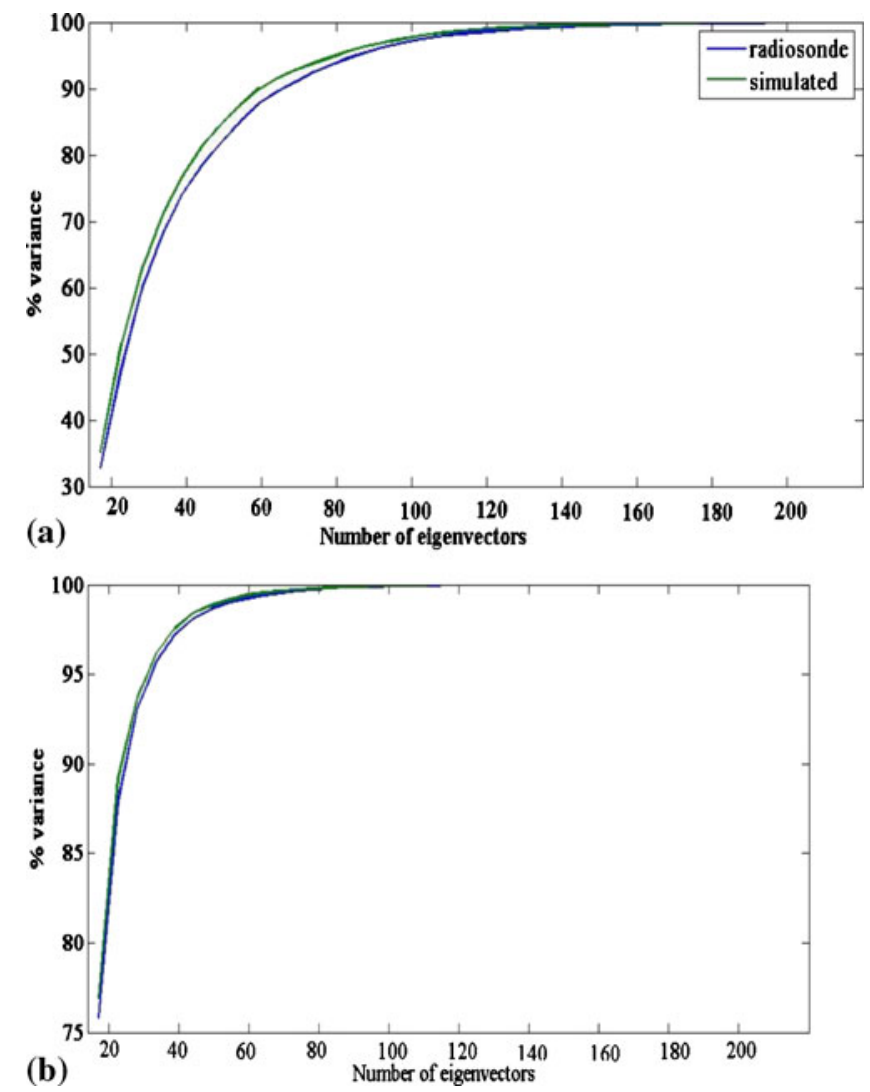

Figure 6. Comparison of $\%$ variance for the synthetic and radiosonde profiles for (a) temperature and (b) humidity. 
profile evaluated at an altitude $z_{k}, \lambda_{v}^{T}$ are the eigen values and $\zeta_{v}$ are non-zero mean Gaussian random numbers with unit variance.

Humidity profiles are generated in a similar way. However, in contrast to temperature, excursions in the humidity can be very large, at times larger than the mean. This can pose a problem because it can lead to negative values which are not realistic. To circumvent this problem, the following transformation proposed by Tatarskaia et al. (1997) is used:

$$
q=\ln a,
$$

where $a$ is the normalized absolute humidity (absolute humidity/maximum humidity across the height for the profile under consideration). The EOF decomposition procedure for humidity is then identical to temperature but $q$ is used instead of $a$. At the final step, we simply invert the results using equation (9), i.e.,

$$
a\left(z_{k}\right)=\exp \left\{\left\langle\ln a\left(z_{k}\right)\right\rangle+\sum_{v=1}^{96} \zeta_{v} \phi_{v}^{\ln a}\left(z_{k}\right) \sqrt{\lambda_{v}^{\ln a}}\right\}
$$

It was seen that the statistics of the 10,000 synthetic profiles generated are consistent with the corresponding statistics of the initial dataset. Figure $6(\mathrm{a}$ and $\mathrm{b})$ shows the comparison of the
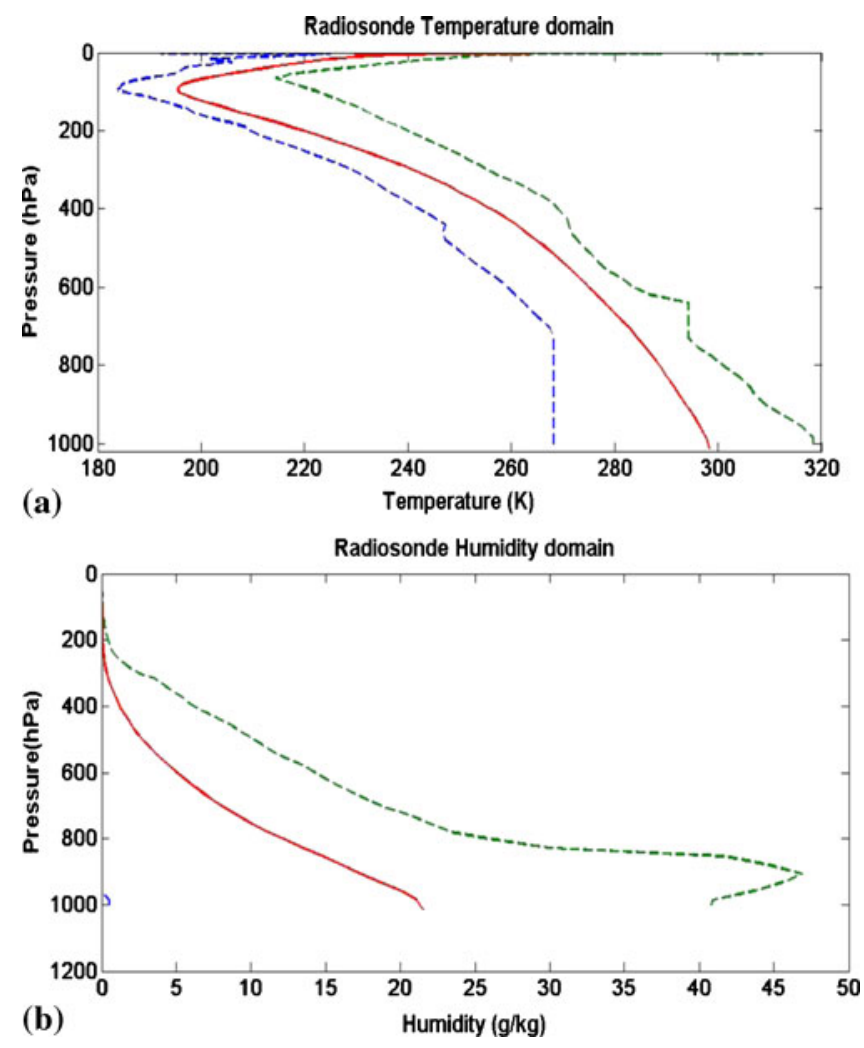

Figure 7. Limiting range for (a) radiosonde temperature and (b) radiosonde humidity.
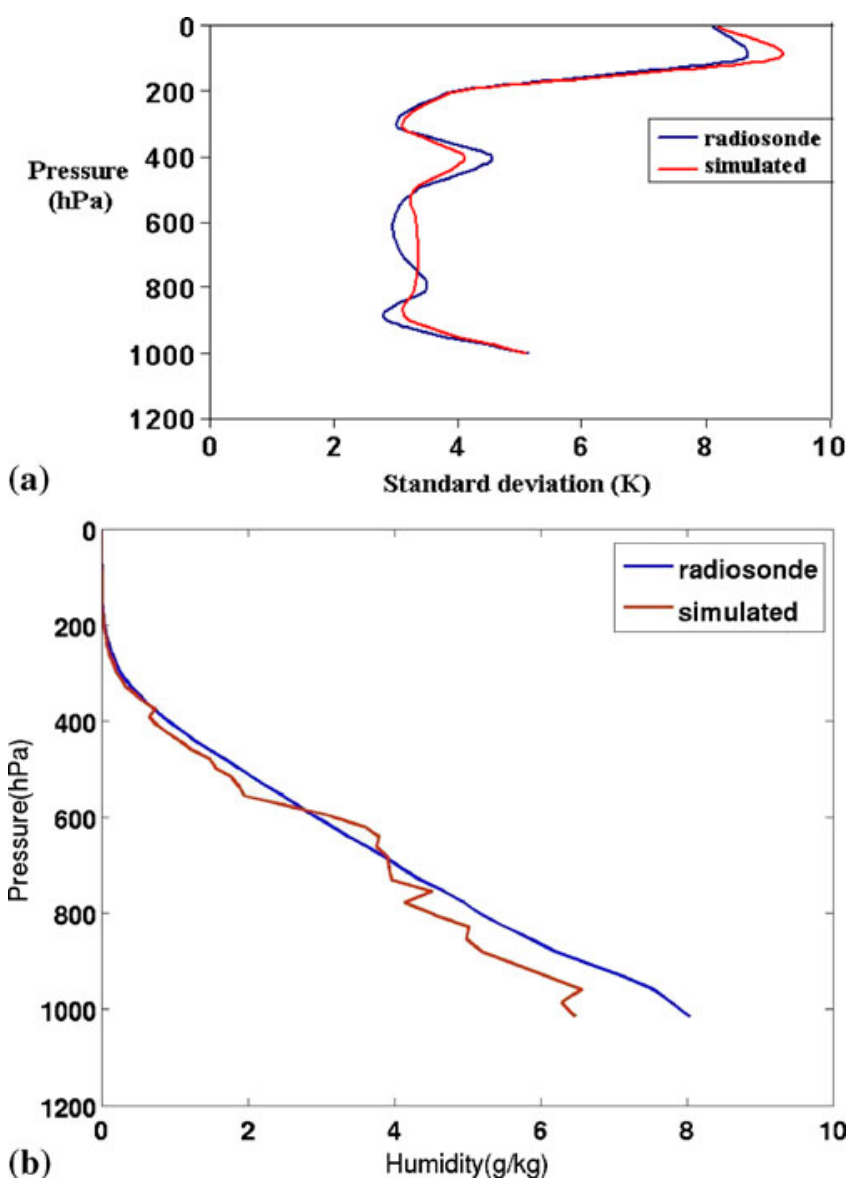

Figure 8. Comparison of standard deviation of radiosonde and synthetic profiles for (a) temperature and (b) humidity.

\%variance explained by the dataset of the temperature and humidity respectively across various pressure levels for the synthetic and the realistic database. Figure 7(a and b) compares the domain of the radiosonde profiles generated for both the temperature and humidity respectively. Figure 8(a and b) shows the comparison between the standard deviations for radiosonde and synthetic profiles for both temperature and humidity. It can be seen that the cumulative variance of the numerically generated parameters agrees well with those of the initial dataset. Furthermore, the mean and the standard deviation of the synthetic profiles generated agree with the initial dataset with a correlation coefficient of over $99 \%$.

\section{Results and discussion}

The back propagation neural network toolbox available in MATLAB is used for training and simulation of the network. The network is trained for 2500 epochs. Six different architectures have been tried in the present work and the network performances are given in table 2 . The root mean 
Table 2. Network performance of various architectures.

\begin{tabular}{lcc}
\hline ANN architecture & RMSE $(K)$ & $\mathrm{R}^{2}$ \\
\hline $193-10-15$ & 2.663 & 0.999 \\
$\mathbf{1 9 3 - 1 3 - 1 5}$ & $\mathbf{1 . 3 9 6}$ & $\mathbf{0 . 9 9 9}$ \\
$193-20-15$ & 2.832 & 0.999 \\
$193-30-15$ & 3.254 & 0.999 \\
$193-40-15$ & 2.868 & 0.999 \\
$193-50-15$ & 2.582 & 0.999 \\
\hline
\end{tabular}

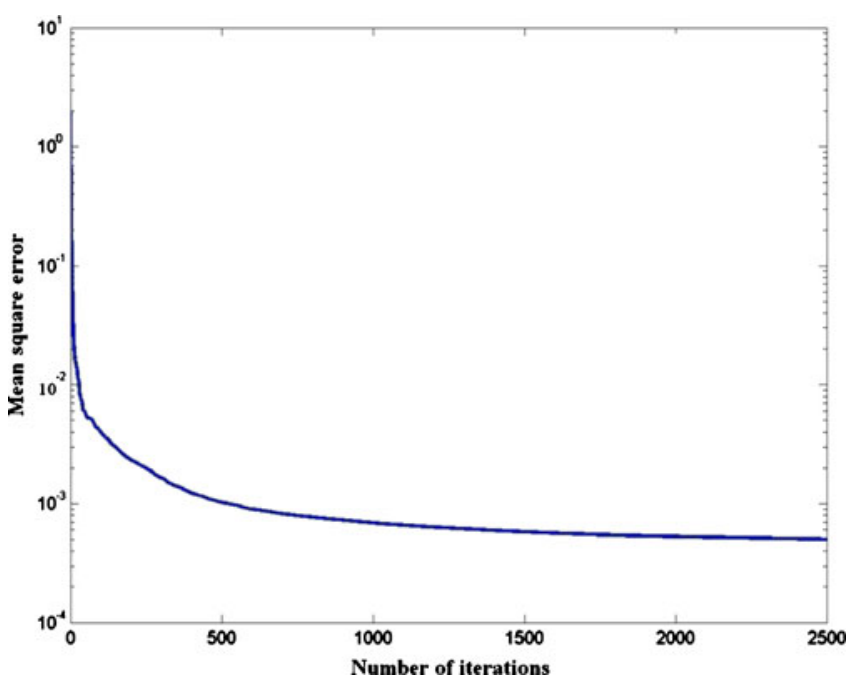

Figure 9. Training performance of the fast forward model.

square error (RMSE) $T_{k}$ at a level $k$ is calculated as:

$$
T_{k}=\sqrt{\frac{\sum_{i=1}^{n}\left(T_{s i}-T_{a i}\right)^{2}}{n}}
$$

where $T_{s i}$ and $T_{a i}$ correspond to the simulated and actual brightness temperatures respectively and $n$ corresponds to the number of profiles in the testing dataset.

From table 2, it can be seen that the architecture with 13 neurons in the hidden layer has the least RMSE of $1.3961 \mathrm{~K}$. This network is considered as the 'best' network and hence further simulations are done with 13 neurons in the hidden layer.

Figure 9 shows the variation of the mean square error (MSE) with epochs. It can be seen that the MSE has reached an acceptable value after 2500 epochs and further iterations are not necessary. The network is then tested for the remaining 1200 profiles in the dataset. Figure 10 shows the parity plot of the brightness temperatures. From the figure, it is clear that the brightness temperatures simulated using the proposed fast forward model agree very well with those simulated by kCARTA and are devoid of any bias. Table 3 shows the bias

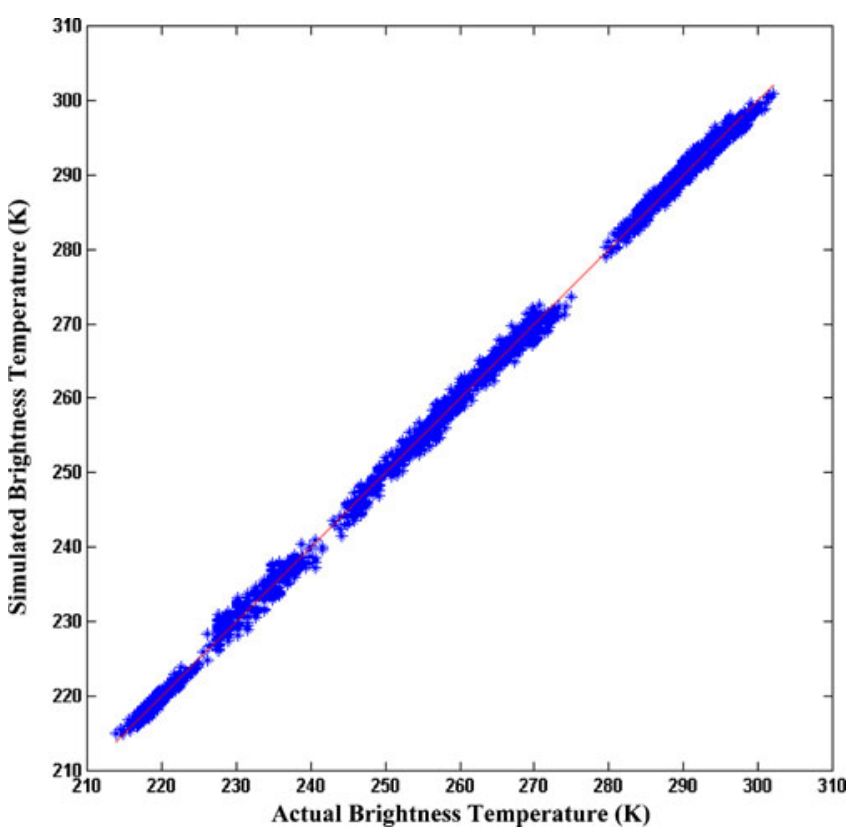

Figure 10. Parity plot between ANN simulated and kCARTA simulated brightness temperatures.

and the RMSE of the brightness temperature simulated at the 15 GOES channels using the fast forward model. It can be seen from table 3 that the accuracy of the fast forward model is about $1.39 \mathrm{~K}$ which confirms the adequacy of the neural network to capture the physics associated with the radiative transfer in the clear sky atmosphere accurately with a correlation coefficient of over 0.99.

Also a comparison of the neural network model with RTTOVv10 has been made for the same set of input profiles. The time taken for computing the radiances of 3876 profiles (with 15 channels) using RTTOVv10 was $0.4 \mathrm{~s}$ (this includes computation time as well as the time taken to write the data to a file) whereas the neural network model was able to complete in $0.165 \mathrm{~s}$. The time taken in RTTOV may vary depending on the input profile used for analysis. Profiles with temperatures and humidity exceeding the limits (as specified in the RTTOV manual) may need extrapolation of the coefficients for computation which may consume time. Also, the 96 pressure levels of kCARTA were interpolated into 51 RTTOV pressure levels using a cubic interpolation method. The RTTOV radiances are based on this 51 level data. The RMSE shown in table 3 was calculated with kCARTA radiances as the reference model and with the same profiles as input.

In order to analyze the robustness of the model, the 'training set' was expanded systematically in steps of 4000 profiles by adding the synthetic profiles numerically generated using EOF. 
Table 3. RMSE of brightness temperatures for various channels.

\begin{tabular}{lcccc}
\hline $\begin{array}{l}\text { Channel } \\
\text { no. }\end{array}$ & $\begin{array}{r}\text { Wavelength } \\
(\mu \mathrm{m})\end{array}$ & $\begin{array}{c}\text { Wavenumber } \\
\left(\mathrm{cm}^{-1}\right)\end{array}$ & $\begin{array}{c}\text { RMSE } \\
(\mathrm{K})\end{array}$ & $\begin{array}{c}\text { Bias } \\
(\mathrm{K})\end{array}$ \\
\hline 1 & 14.71 & 680 & 0.557 & 0.00215 \\
2 & 14.37 & 696 & 0.406 & $7.64 \mathrm{E}-05$ \\
3 & 14.06 & 711 & 0.483 & -0.00023 \\
4 & 13.96 & 733 & 0.409 & -0.00077 \\
5 & 13.37 & 748 & 0.415 & -0.0018 \\
6 & 12.66 & 790 & 0.821 & -0.00111 \\
7 & 12.02 & 832 & 0.889 & -0.0005 \\
8 & 11.03 & 907 & 0.712 & 0.001023 \\
9 & 9.71 & 1030 & 1.265 & -0.00181 \\
10 & 7.43 & 1345 & 0.942 & 0.000452 \\
11 & 7.02 & 1425 & 1.039 & -0.00186 \\
12 & 6.51 & 1532 & 1.396 & -0.00205 \\
13 & 4.57 & 2188 & 0.781 & 0.000418 \\
14 & 4.52 & 2213 & 0.739 & 0.000316 \\
15 & 4.45 & 2247 & 0.479 & $-3.62 \mathrm{E}-06$ \\
\hline
\end{tabular}

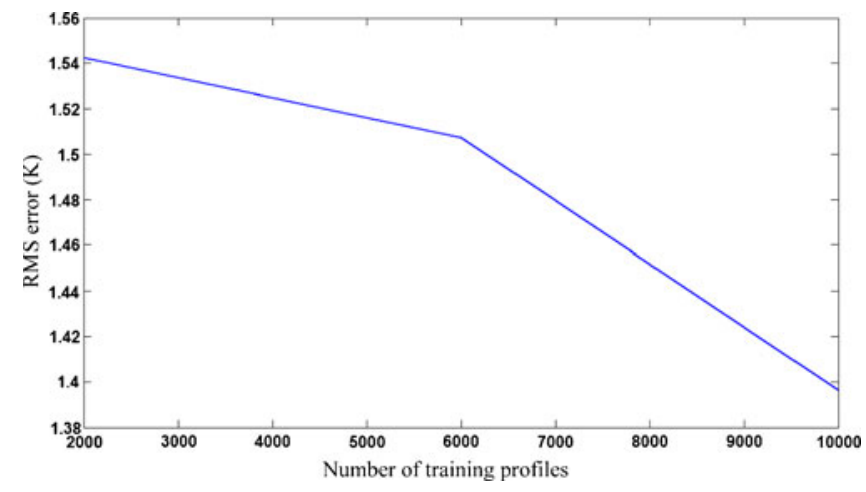

Figure 11. Variation of RMS error (K) with increase in the number of training profiles.

To validate the accuracy of the EOF in generating synthetic profiles, a sample of 100 profiles were generated using the method of EOF. kCARTA was then used to simulate the radiances for these 100 profiles. These radiances simulated were then compared with the radiances generated for the same profiles using the proposed fast forward model. It was found that the two quantities matched very well with a correlation coefficient of over 0.999, thus confirming the accuracy of the method.

Figure 11 shows the variation of the RMSE with the expansion of the training set. Although there was negligible difference in the maximum RMSE with the addition of profiles to the training set, the RMSE reduces marginally, thereby confirming the robustness of the proposed model. More importantly, it confirms that the original database employed itself contains enough diversity to capture all of the physics and injection of additional data improves the already good results only marginally.
Furthermore, the execution of the forward model on an Intel Xeon 3.0 GHz processor based, $16 \mathrm{~GB}$ DDR2 RAM server for 3876 test profiles took $59 \mathrm{~h}$ using kCARTA, while the fast forward model delivers the same results in just $3 \mathrm{~s}$. Hence, the fast forward model can be successfully employed for developing quicker and accurate retrieval algorithms.

\section{Conclusions}

A neural network based fast forward radiative transfer model for the 19 channel infrared sounder of the proposed Indian satellite INSAT 3D has been developed in the present work. The fast model is proposed as a replacement for the 'full' radiative transfer (RT) simulations after elaborate testing and benchmarking. The wavenumbers considered for the channels in this study correspond to those of the GOES sounder, which are identical to that of INSAT 3D. A large database of atmospheric temperature and humidity profiles against the multispectral radiances is first generated using the kCARTA to simulate the radiances for the given atmospheric temperature and humidity profiles. This database powers the training of the neural network. Additional profiles not used in the training were used for testing. The maximum value of the RMSE on the brightness temperature was $1.39 \mathrm{~K}$ which confirms the adequacy of the neural network to capture the physics associated with the radiative transfer in the clear sky atmosphere accurately with a correlation coefficient of over $99 \%$. Finally, in order to establish the robustness of the model, 10,000 synthetic profiles are generated using the method of empirical orthogonal functions. 
Keeping the testing set the same, the training set was then systematically expanded in steps of 4000 profiles by adding these synthetic profiles generated. The results obtained are very encouraging and show that the fast forward model using the neural network is very robust and is capable of predicting the brightness temperatures accurately with a correlation coefficient of over $99 \%$ in just a fraction of the time required by the detailed numerical simulations. The proposed fast forward model can be suitably fine tuned when the satellite is operational and can be used by the community to develop online retrieval algorithms.

\section{References}

Andrieu C and Arnaud Doucet 2000 Simulated annealing for maximum a posteriori parameter estimation of hidden markov models; IEEE Trans. Inform. Theor. 46(3) 9941004.

Blackwell W J 2005 A neural-network technique for the retrieval of atmospheric temperature and moisture profiles from high spectral resolution sounding data; IEEE Trans. Geosci. Rem. Sens. 43(11) 2535-2546.

Crewell S, Ulrich Löhnert and Thomas Rose 2002 Synergetic algorithms for temperature and humidity profiling based on ground-based microwave measurements; First COST 720 Workshop, Italy.

Cooperative Institute for Meteorological Satellite Studies (CIMSS) GOES-13 Science Report, 2006, URL: http:// cimss.ssec.wisc.edu/goes/g13_report/.

Machado De Souza S, Strow L L, Motteler H E, Benson R G and Hannon S E 1998 Fast computation of monochromatic infrared atmospheric atmospheric transmittances using compressed look-up tables; $J$.
Quantitative Spectroscopy and Radiative Transfer $\mathbf{2 8 3 0}$ 481-493.

Donald Hillger W and Timothy Schmit J 2007 The GOES13 Science Test: Imager and Sounder radiance and Product validations, National Oceanic and Atmospheric Administration (NOAA) Technical Report NESDIS 125, $13 \mathrm{p}$.

Jiang D, Chaohua Dong, Peng Zhang and Xuebao Wu 2005 Neural networks for atmospheric temperature retrieval from AQUA AIRS/AMSU/HSB measurements; ITSC XIV Proceedings, China.

Katti V R, Pratap V R, Dave R K and Mankad K N 2006 INSAT-3D: An advanced meteorological mission over Indian ocean; Proceedings of SPIE, Int. Soc. Optical Engineering 6407 640-709.

Liou K N, Ou S C and King J F 1992 An assessment of the differential inversion method for remote sounding of temperatures; J. Appl. Meteorol. 32 1108-1123.

Li Jun 1994 Temperature and water vapor weighting functions from radiative transfer equation with surface emissivity and solar reflectivity; Adv. Atmos. Sci. 11 421-426.

Tatarskaia Maia S, Richard Lataitis J and Boba Stankov B 1997 A numerical method for synthesizing atmospheric temperature and humidity profiles; J. Appl. Meteorol. 37 $718-729$.

Smith W L, Woolf H M and Fleming H E 1972 Retrieval of atmospheric temperature profiles from satellite measurements and dynamic forecasting; J. Appl. Meteorol. 11 $113-122$.

Suggs R J, Stephanie Haines L and Gary Jedlovec J 2003 Land surface temperature retrievals from GOES- 8 using emissivities retrieved from MODIS; 12th Conference on Satellite Meteorology and Oceanography, California.

Sun D, Pinker R T and Basara J B 2004 Land surface temperature estimation from the next generation of geostationary operational environmental satellites: GOES M-Q; J. Appl. Meteorol. 43 363-372.

Yeh H M, Vonder Haar T H and Liou K N 1985 Cloud parameters and temperature profile retrieval from Infrared sounder data; J. Atmos. Sci. 42 2360-2370. 